\title{
$B R A F$ mutations as a therapeutic target in metastatic colorectal cancer patients: a long due success
}

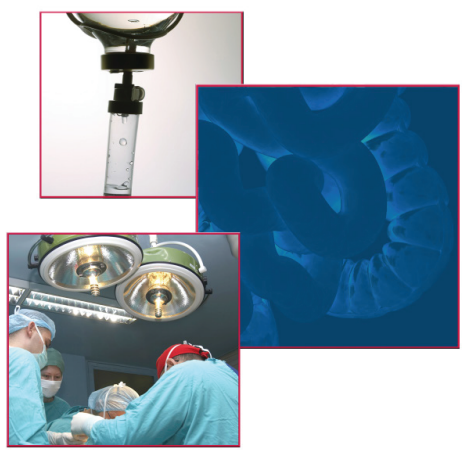

\author{
Ibrahim Halil Sahin*, 1(iD) \& Jim Klostergaard ${ }^{2}$ \\ ${ }^{1}$ Emory University School of Medicine, Winship Cancer Institute, Atlanta, GA, USA \\ ${ }^{2}$ Department of Molecular \& Cellular Oncology, MD Anderson Cancer Center, Houston, TX, USA \\ *Author for correspondence: ihsahin@emory.edu
}

\begin{abstract}
'The triple combination of dabrafenib, trametinib and panitumumab led to improved clinical responses with an ORR of $21 \%$ as compared with the combination of dabrafenib and panitumumab (an ORR of 10\%) or trametinib and panitumumab (no objective responses)"
\end{abstract}

First draft submitted: 24 September 2019; Accepted for publication: 27 September 2019; Published online: 12 November 2019

Keywords: binimetinib • BRAF • colorectal cancer $(C R C) \bullet$ dabrafenib • encorafenib • immunotherapy • MEK • $\mathrm{MSI}-\mathrm{H} \bullet$ trametinib $\bullet$ vemurafenib

Colorectal cancer (CRC) continues to be a challenging cancer with an increasing incidence among young adults, which has led to changes in recommendations for screening. Consistent with this trend, CRC remains the third leading cause of cancer-related death in the USA among men and women [1]. Oncogenic driver genes among young adults appear to be different; perhaps this is associated with their distinct molecular behavior and has a resultant direct effect on the clinical course of the disease [2]. The rising incidence of young-onset advanced-stage CRC will also likely impact the molecular landscape of CRC as well as the frequency of potentially actionable molecular alterations.

Recent progress in the management of metastatic CRC patients, particularly in the field of precision medicine, will likely enhance our therapeutic options and lead to significant improvements in the outcomes of advanced-stage patients. With the use of next-generation sequencing, comprehensive molecular profiling can be conducted with relatively reasonable tumor sample sizes and within reasonable time durations ( $\sim 4$ weeks); this can potentially reveal actionable molecular alterations that are targetable, such as mismatch repair deficiency (MMR-D), HER2 amplification and BRAF V600 mutations.

$B R A F$ V600 mutations have long been of interest in the field of targeted therapeutics. Recently, combined approaches with EGFR blockade and $B R A F$ inhibitors, with and without $M E K$ inhibitors, have resulted in promising clinical outcomes. In this article, we elaborate on the clinical and molecular characteristics of BRAFV600 mutations in the CRC patient and discuss recent clinical data from early phase clinical trials investigating the therapeutic targeting of BRAF V600 mutations.

\section{Oncogenic $B R A F$ mutations at a glance}

$B R A F$ is a well-established oncogene and gain of function mutations have been identified in multiple solid tumors, including melanoma, lung and colorectal cancers [3]. $B R A F$ is a member of the RAF kinase family (a serine/threonineprotein kinase family) and it has a key function in the MAP kinase signaling pathway which drives cellular growth and proliferation [4]. In the MAP kinase pathway, $B R A F$ is regulated by upstream $R A S$ signaling, which is, in turn, activated by receptor tyrosine kinases such as EGFR [5].

$B R A F$ V600E mutation, which is by far the most common (80-97\%) among BRAF mutations in CRC patients, results in substitution of glutamic acid (E) for valine $(\mathrm{V})$ at position 600 in the kinase domain of the protein [3,6]. Other less common BRAF mutations, including V600K, V600R, K601E and G469A, have been shown to be involved in carcinogenesis in multiple solid tumors, including CRC [7,8]. However, it is important to note that their

Future $\because$ Medicine 
oncogenic activity is significantly different [9] when compared with $B R A F$ V600E. For example, in one preclinical study analyzing the $B R A F$ mutations in vitro, the authors demonstrated higher oncogenic $E R K$ pathway activation with $B R A F$ V600E mutation as compared with $B R A F$ V600K mutation and even more interestingly, decreased $B R A F$ activity and $E R K$ pathway activation with certain mutations such as $B R A F \mathrm{D} 594 \mathrm{~V}$ [9]. These data suggest that the clinical significances of $B R A F$ mutations should be carefully assessed in each clinical scenario based on the specific mutation, rather than conceptually considering all $B R A F$ mutations in a single, equivalent category. The mutations causing an oncogenic gain of function in the $B R A F$ gene result in a constitutively-activated downstream $M E K-E R K$ signaling pathway, thus inducing cell growth, proliferation and invasion $[8,10]$.

\section{Clinical characteristics of BRAF mutations in metastatic colorectal cancer patients}

$B R A F$ mutations have been identified in approximately $5-10 \%$ of CRC patients in different patient cohorts $[11,12]$. The frequency of $B R A F$ mutation is greater in tumors with microsatellite instability (MSI) as compared with microsatellite stable (MSS) tumors, particularly in sporadic microsatellite unstable tumors that are associated with CpG island methylator phenotype [13-15]. Multiple studies have suggested worse clinical outcomes in CRC patients with a $B R A F$ mutation, in particular with MSS tumors [13]. For example, in one clinical cohort study, the authors showed $B R A F$ mutations may be one of the predictors of worse outcomes in early-stage CRC patients who underwent surgical resections [16]. Similarly, in metastatic disease, tumors with BRAFV600E mutation behave aggressively and lead to early disease progression $[17,18]$. Notably, $B R A F$-mutant CRC tumors with MSI appear to have better outcomes when compared with MSS patients, indicating a distinct clinical behavior, perhaps related to the immunogenic nature of tumors with MSI tumors being infiltrated by tumor-reactive lymphocytes $[13,19]$. Therefore, CRC patients with BRAF mutations should be evaluated based on their MSI status and the clinical approach to therapeutic options should be determined in a different manner as discussed below. Notably, MSI itself may result in uncommon BRAF mutations (unlike BRAF V600E driven CpG island methylator phenotype tumors) as a consequence of frameshift mutations, but their clinical significances are currently unclear.

\section{Clinical trials investigating the efficacy of BRAF targeting agents in combination with EGFR blockade}

$B R A F$ mutations have been a focus for investigators as a potential target given its biological and clinical significance. Promising response rates and improvement in clinical outcomes in melanoma patients with single-agent vemurafenib, a $B R A F$ inhibitor, have not been translated into clinically meaningful effects in CRC patients [20]. Unlike melanoma, CRC carries different molecular characteristics, with various alternative oncogenic pathways for activation that serve as escape mechanisms against BRAF inhibitors. For example, a landmark preclinical study in BRAF-mutant CRC showed rebound activation of EGFR signaling in the presence of BRAF inhibition [21]. Consistent with preclinical data, outcomes with single-agent $B R A F$ inhibitors, in $B R A F$-mutant CRC, did not lead to significant clinical responses [20,22]. These findings have led to clinical trials combining $B R A F$ inhibitors and EGFR blockade.

In an early-phase clinical trial, vemurafenib was combined with irinotecan $\left(180 \mathrm{mg} / \mathrm{m}^{2}\right)$ and an EGFR monoclonal antibody, cetuximab $\left(500 \mathrm{mg} / \mathrm{m}^{2}\right)$ known as VIP regimen. This treatment plan resulted in an objective response rate (ORR) and median progression free survival of $35 \%$ and 7.7 months, respectively, in metastatic BRAF V600E mutant CRC patients who were previously treated with cytotoxic agents [23]. The most common adverse events noted with this triplet therapy were - expectedly - fatigue (89\%), diarrhea (84\%), nausea (79\%) and rash (74\%). In another Phase I trial, dabrafenib (a BRAF inhibitor) and trametinib (an $M E K$ inhibitor) with panitumumab (an EGFR monoclonal antibody) were investigated in previously-treated metastatic CRC patients with a $B R A F$ mutation, for both safety and early clinical efficacy analysis [24]. The triple combination of dabrafenib, trametinib and panitumumab led to improved clinical responses with an ORR of $21 \%$ as compared with the combination of dabrafenib and panitumumab (an ORR of 10\%) or trametinib and panitumumab (no objective responses).

The safety lead-in of a Phase III trial (the BEACON trial) investigated the combination of encorafenib (a $B R A F$ inhibitor) and binimetinib (a $M E K$ inhibitor) with cetuximab in previously treated metastatic $B R A F$-mutant CRC patients [25]. In this study, patients received triplet therapy as a combination of encorafenib (300 mg daily), binimetinib ( $45 \mathrm{mg}$ twice daily) and cetuximab at $400 \mathrm{mg} / \mathrm{m}^{2}$ (first dose) followed by $250 \mathrm{mg} / \mathrm{m}^{2}$ per week as a 28 -day cycle regimen. The authors reported very promising ORR (41\%) although only a limited number of patients were evaluated at the time of the report (a total of 29 patients). The authors also reported that an additional nine 
patients had durable disease control, further indicating promising therapeutic efficacy. Based on these findings, the US FDA granted breakthrough therapy designation to this triplet therapy [26]. In this study, the toxicities related to this triplet therapy were manageable and the most common adverse events were fatigue (14\%), anemia (10\%), increased CPK (10\%) and AST (10\%). Most recently, the authors reported the final results of the safety lead-in part of this trial and identified a progression free survival and overall survival of 8.0 and 15.3 months, respectively [27]. At this time, National Comprehensive Cancer Network guidelines have been updated for these different approaches targeting $B R A F$ mutations in metastatic CRC patients [26].

It is important to note that the therapeutic approach for $B R A F$-mutant CRC with MSI-High (MSI-H) disease should be different. BRAF-mutant CRC patients with MMR-D/MSI-H disease should be considered for immune checkpoint inhibitor-based therapy, which includes nivolumab, nivolumab with ipilimumab or pembrolizumab, based on the recent evidence with promising clinical responses [28-30]. Therefore, the MMR-D/MSI-H should be evaluated in all $B R A F$-mutant CRC patients, if they have not already been assessed at the time of initial diagnosis.

\section{Conclusion \& future perspective}

With the recent progress in targeted cancer therapeutics, the combination approaches targeting $B R A F$ mutations and associated feedback loops have yielded significant clinical responses; in fact, one of these combinations (encorafenib, binimetinib and cetuximab) has been granted breakthrough therapy designation, based on the promising results in early reports of the BEACON trial $[27,31]$. The other aforementioned combinations have also been implemented in National Comprehensive Cancer Network guidelines and can be considered in BRAF-mutant CRC patients. Perhaps in the near future, all the aforementioned combined therapeutic options targeting BRAF mutations in CRC patients will likely be approved by the FDA. However, it is important to note that, the studies discussed above have evaluated these patients in chemotherapy-refractory settings, and therefore these agents should be used as a subsequent line of therapy based on the current evidence. In the first-line setting, FOLFOXIRI in combination with bevacizumab should be considered in patients with good performance status [32]. Notably, upfront classification of $B R A F$-mutant CRC patients based on their MMR-D/MSI-H status is required, as patients with MMR-D/MSI-H disease in the setting of a $B R A F$ mutation may benefit from immune checkpoint inhibitors (pembrolizumab, nivolumab or nivolumab with ipilimumab) and achieve durable clinical responses [28,29]. It is also important to note that these agents have already been approved by the FDA for this subset of CRC patients.

Even though these promising results will likely improve the outcomes in this aggressive subset of CRC patients, there are resistance mechanisms that develop progressively. In a translational study, resistance mechanisms were investigated in patients with $B R A F$-mutant CRC treated with BRAF/MEK inhibitors and multiple aberrations were identified that reconstituted MAP kinase signaling, including BRAF and $K R A S$ amplification and a MEK1 mutation [33]. These data suggest that additional blockade(s) downstream of the MAP kinase pathway could potentially further enhance the efficacy of $B R A F$-targeting agents and may prevent development of resistance that can be attributed to MAP kinase signaling. Perhaps performing molecular profiling of circulating tumor DNA upon progression may advance our understanding related to mechanisms of acquired resistance. Moreover, molecular profiling may potentially uncover other actionable genes that can be targeted in a clinical trial setting. As discussed above, the significance of other uncommon BRAF mutations (besides BRAF V600E and BRAF V600K) in CRC patients is unknown and may or may not be related to the driver gene. Interestingly, a recent study reported the benefit of the use of EGFR-directed therapies without BRAF inhibitors in CRC patients with BRAF non-V600 mutations [34]. This recent finding suggests that $B R A F$ mutations should not be lumped into a single category and each case should be evaluated individually based on the class of $B R A F$ mutation [9].

Detection of other clinically relevant genomic alterations in CRC patients may also provide important data regarding other driver genes, e.g., KRAS, PIK3CA and HER2. Therefore, we propose that all BRAF-mutant metastatic CRC patients should undergo next-generation based molecular profiling for better characterization of the molecular signature of the tumor and to best determine the therapeutic actions.

Financial \& competing interests disclosure

The authors have no relevant affiliations or financial involvement with any organization or entity with a financial interest in or financial conflict with the subject matter or materials discussed in the manuscript. This includes employment, consultancies, honoraria, stock ownership or options, expert testimony, grants or patents received or pending, or royalties.

No writing assistance was utilized in the production of this manuscript. 


\section{Open access}

This work is licensed under the Attribution-NonCommercial-NoDerivatives 4.0 Unported License. To view a copy of this license, visit http://creativecommons.org/licenses/by-nc-nd/4.0/

\section{References}

1. Siegel RL, Miller KD, Jemal A. Cancer statistics, 2019. CA Cancer J. Clin. 69(1), 7-34 (2019).

2. Tricoli JV, Blair DG, Anders CK et al. Biologic and clinical characteristics of adolescent and young adult cancers: acute lymphoblastic leukemia, colorectal cancer, breast cancer, melanoma, and sarcoma. Cancer 122(7), 1017-1028 (2016).

3. Davies H, Bignell GR, Cox C et al. Mutations of the BRAF gene in human cancer. Nature 417(6892), 949 (2002).

4. Tuveson DA, Weber BL, Herlyn M. BRAF as a potential therapeutic target in melanoma and other malignancies. Cancer Cell 4(2), 95-98 (2003).

5. Haluska F, Pemberton T, Ibrahim N, Kalinsky K. The RTK/RAS/BRAF/PI3K pathways in melanoma: biology, small molecule inhibitors, and potential applications. Semin. Oncol. 34(6), 546-54 (2007).

6. Bamford S, Dawson E, Forbes S et al. The COSMIC (Catalogue of Somatic Mutations in Cancer) database and website. Br. J. Cancer 91(2), 355 (2004).

7. Flaherty KT, Mcarthur G. BRAF, a target in melanoma: implications for solid tumor drug development. Cancer 116(21), 4902-4913 (2010).

8. Thiel A, Ristimäki A. Toward a molecular classification of colorectal cancer: the role of BRAF. Front. Oncol. 3, 281 (2013).

9. Wan PT, Garnett MJ, Roe SM et al. Mechanism of activation of the RAF-ERK signaling pathway by oncogenic mutations of B-RAF. Cell 116(6), 855-867 (2004).

10. Brennan DF, Dar AC, Hertz NT et al. A Raf-induced allosteric transition of KSR stimulates phosphorylation of MEK. Nature 472(7343), 366 (2011).

11. Vaughn CP, Zobell SD, Furtado LV, Baker CL, Samowitz WS. Frequency of KRAS, BRAF, and NRAS mutations in colorectal cancer. Genes Chromosomes Cancer 50(5), 307-312 (2011).

12. Rajagopalan H, Bardelli A, Lengauer C, Kinzler KW, Vogelstein B, Velculescu VE. Tumorigenesis: $R A F / R A S$ oncogenes and mismatch-repair status. Nature 418(6901), 934 (2002).

13. Samowitz WS, Sweeney C, Herrick J et al. Poor survival associated with the BRAF V600E mutation in microsatellite-stable colon cancers. Cancer Res. 65(14), 6063-6069 (2005).

14. Deng G, Bell I, Crawley S et al. BRAF mutation is frequently present in sporadic colorectal cancer with methylated hMLH1, but not in hereditary nonpolyposis colorectal cancer. Clin. Cancer Res. 10(1), 191-195 (2004).

15. Weisenberger DJ, Siegmund KD, Campan M et al. CpG island methylator phenotype underlies sporadic microsatellite instability and is tightly associated with BRAF mutation in colorectal cancer. Nat. Genet. 38(7), 787 (2006).

16. Fariña-Sarasqueta AV, van Lijnschoten G, Moerland E et al. The BRAF V600E mutation is an independent prognostic factor for survival in stage II and stage III colon cancer patients. Ann. Oncol. 21(12), 2396-2402 (2010).

17. Richman SD, Seymour MT, Chambers $\mathrm{P}$ et al. KRAS and BRAF mutations in advanced colorectal cancer are associated with poor prognosis but do not preclude benefit from oxaliplatin or irinotecan: results from the MRC FOCUS trial. J. Clin. Oncol. 27(35), 5931-5937 (2009).

18. Yokota T, Ura T, Shibata $\mathrm{N}$ et al. BRAF mutation is a powerful prognostic factor in advanced and recurrent colorectal cancer. Br. J. Cancer 104(5), 856 (2011).

19. Rosenbaum MW, Bledsoe JR, Morales-Oyarvide V, Huynh TG, Mino-Kenudson M. PD-L1 expression in colorectal cancer is associated with microsatellite instability, BRAF mutation, medullary morphology and cytotoxic tumor-infiltrating lymphocytes. Mod. Pathol. 29(9), 1104 (2016).

20. Hyman DM, Puzanov I, Subbiah V et al. Vemurafenib in multiple nonmelanoma cancers with BRAF V600 mutations. N. Engl. J. Med. 373(8), 726-736 (2015).

21. Prahallad A, Sun C, Huang S et al. Unresponsiveness of colon cancer to BRAF (V600E) inhibition through feedback activation of EGFR. Nature 483(7387), 100 (2012).

22. Kopetz S, Desai J, Chan E et al. PLX4032 in metastatic colorectal cancer patients with mutant BRAF tumors. J. Clin. Oncol. 28(Suppl. 15), 3534-3534 (2010).

23. Hong DS, Morris VK, El Osta B et al. Phase IB study of vemurafenib in combination with irinotecan and cetuximab in patients with metastatic colorectal cancer with BRAFV600E mutation. Cancer Discov. 6(12), 1352-1365 (2016).

24. Corcoran RB, André T, Atreya CE et al. Combined BRAF, EGFR, and MEK inhibition in patients with BRAFV600E-mutant colorectal cancer. Cancer Discov. 8(4), 428-443 (2018).

25. Van Cutsem E, Cuyle P-J, Huijberts S et al. BEACON CRC study safety lead-in (SLI) in patients with BRAF V600E metastatic colorectal cancer (mCRC): efficacy and tumor markers. J. Clin. Oncol. 36(4), 627 (2018). 
26. Benson AB, Venook AP, Al-Hawary MM et al. NCCN guidelines insights: colon cancer, version 2.2018. J. Natl Compr. Canc. Netw. 16(4), 359-369 (2018).

27. Van Cutsem E, Huijberts S, Grothey A et al. Binimetinib, encorafenib, and cetuximab triplet therapy for patients with BRAF V600E-mutant metastatic colorectal cancer: safety lead-in results from the Phase III BEACON colorectal cancer study. J. Clin. Oncol. 37(17), 1460-1469 (2019).

28. Le DT, Uram JN, Wang H et al. PD-1 blockade in tumors with mismatch-repair deficiency. N. Engl. J. Med. 372(26), 2509-2520 (2015).

29. Overman MJ, Lonardi S, Wong KYM et al. Durable clinical benefit with nivolumab plus ipilimumab in DNA mismatch repair-deficient/microsatellite instability-high metastatic colorectal cancer. J. Clin. Oncol. 36(8), 773-779 (2018).

30. Overman MJ, Mcdermott R, Leach JL et al. Nivolumab in patients with metastatic DNA mismatch repair-deficient or microsatellite instability-high colorectal cancer (CheckMate 142): an open-label, multicentre, Phase 2 study. Lancet Oncol. 18(9), 1182-1191 (2017).

31. Jones D. Pierre Fabre: and its partner array BioPharma announce 15.3 months median OS observed from the Phase 3 BEACON CRC safety lead-in of BRAFTOVI ${ }^{\circledR}$, MEKTOVI $^{\circledR}$ and ERBITUX ${ }^{\circledR}$ in BRAF-mutant metastatic CRC (2019). https://www.pierre-fabre.com/en/press_release/pierre-fabre-and-its-partner-array-biopharma-announce-study-results

32. Loupakis F, Cremolini C, Salvatore L et al. FOLFOXIRI plus bevacizumab as first-line treatment in BRAF mutant metastatic colorectal cancer. Eur. J. Cancer 50(1), 57-63 (2014).

33. Ahronian LG, Sennott EM, Van Allen EM et al. Clinical acquired resistance to RAF inhibitor combinations in BRAF-mutant colorectal cancer through MAPK pathway alterations. Cancer Discov. 5(4), 358-367 (2015).

34. Yaeger R, Kotani D, Mondaca S et al. Response to anti-EGFR therapy in patients with BRAF non-V600-mutant metastatic colorectal cancer. Clin. Cancer Res. (2019). 10.1158/1078-0432 